OPEN

SUBJECT AREAS:

CRYOELECTRON

MICROSCOPY

ION CHANNELS IN THE NERVOUS SYSTEM

Received

10 September 2014

Accepted

20 October 2014

Published

18 November 2014

Correspondence and requests for materials should be addressed to J.R.M. (joel. meyerson@nih.gov) or S.S. (ss1@nih.gov)

\section{Self-assembled monolayers improve protein distribution on holey carbon cryo-EM supports}

Joel R. Meyerson' ', Prashant Rao', Janesh Kumar'², Sagar Chittori², Soojay Banerjee', Jason Pierson ${ }^{3}$, Mark L. Mayer ${ }^{2} \&$ Sriram Subramaniam

'Laboratory of Cell Biology, Center for Cancer Research, NCI, NIH, Bethesda, MD 20892, ${ }^{2}$ Laboratory of Cellular and Molecular
Neurophysiology, Porter Neuroscience Research Center, NICHD, NIH, Bethesda MD 20892, ${ }^{3}$ FEI Company, Hillsboro, OR 97124.

Poor partitioning of macromolecules into the holes of holey carbon support grids frequently limits structural determination by single particle cryo-electron microscopy (cryo-EM). Here, we present a method to deposit, on gold-coated carbon grids, a self-assembled monolayer whose surface properties can be controlled by chemical modification. We demonstrate the utility of this approach to drive partitioning of ionotropic glutamate receptors into the holes, thereby enabling 3D structural analysis using cryo-EM methods.

T

hree-dimensional cryo-electron microscopy (cryo-EM) has experienced dramatic growth over the past decade as evidenced by the rapid rise in high quality macromolecular structures reported ${ }^{1}$. Though in practice, X-ray crystallography usually provides higher resolution protein structural information in the 1.0-4.0 ̊ range, single particle cryo-EM is now capable of achieving resolutions as high as $3.2 \AA$, even for protein assemblies smaller in size than $500 \mathrm{kDa}^{2,3}$. The ability to determine structures at high resolution from native proteins purified to modest concentrations $(1-2 \mathrm{mg} / \mathrm{mL})$ provides the opportunity to study important classes of macromolecules including membrane proteins that can often be challenging targets for X-ray crystallography because of difficulties associated with obtaining suitable, well-ordered crystals.

Though cryo-EM software and hardware technologies have developed significantly, the methods used to prepare specimens for cryo-EM have not changed appreciably in the past three decades. Thus, the majority of 3D cryo-EM structures have been determined using methods in which aqueous suspensions of macromolecule are imaged on holey carbon supports, following plunge-freezing in liquid cryogens ${ }^{4}$ or methods by which sugar-embedded or aqueous suspensions of macromolecules are imaged on continuous carbon supports, also following plunge-freezing in liquid cryogens ${ }^{5}$. With the availability of direct electron detectors and next-generation microscopes having more stable optics with constant power lenses, three-condenser optics and aberration correctors, specimen quality is now widely perceived as one of the major limitations for achieving higher resolution in structures determined by cryoEM. A variety of materials and methods have been tested with cryo-EM supports in recent years including ultrathin carbon and graphen ${ }^{6-8}$, silicon carbide ${ }^{9}$, amorphous titanium-silicon glass ${ }^{10}$, and inkjet deposition ${ }^{11}$, but these more exotic approaches have not found widespread adoption because they can require specialized and costly equipment. Moreover, continuous layer supports such as graphene can cause the target molecule to adopt preferred orientations, which may complicate or impede structure determination ${ }^{12}$. Another powerful approach is to use affinity methods to capture target macromolecules using immobilized antibodies or similar binding strategies, but this also is limited to having a continuous substrate such as a layer of carbon film present in the background ${ }^{13}$. Despite all of these alternatives, commercially produced amorphous holey carbon grids remain the preferred cryo-EM support material, because they are relatively inexpensive and easy to use.

The principal goal in specimen preparation with holey carbon film is to achieve a uniform layer of vitrified material where the protein is partitioned in random orientations in the holes. However, this can be difficult to achieve, even after altering hydrophilicity by the commonly used technique of glow discharge ${ }^{14}$. In such cases it can still be possible to obtain high-resolution structural information by using a continuous carbon film rather than holey carbon, provided the molecules do not adopt preferential orientations. For large complexes such as ribosomes, this approach works well, but for small protein complexes the contribution from the carbon support makes it difficult to determine a structure using conventional single particle cryo-EM. One way to mitigate the problem is to 
lower the effect of the background contribution with tomography and sub-volume averaging. We recently used this approach in our lab to study the kainate receptor GluK2, a member of the family of ionotropic glutamate receptors, and obtained 3D structures at $\sim 20 \AA$ resolution ${ }^{15}$.

\section{Results}

In order to obtain higher resolution structures of the kainate receptor using single particle analysis, we sought to reduce the adsorption of GluK2 to the carbon support and improve partitioning of the protein into the holes. To this end we introduced a hydrophilic selfassembled monolayer (SAM) to the standard holey carbon support ${ }^{16}$. This was done by sputter coating both sides of the grid with a gold film, and then reacting the gold-coated grid with a linear thiol bearing a PEG group (Fig. 1). Chemical bonding between the gold surface and the thiol group, results in a surface bearing a layer of PEG groups, which are known to be hydrophilic and passive to biochemical macromolecules ${ }^{16}$. The specificity of the interaction between the thiol and the gold layer makes it more chemically and spatially controlled than the approach of coating the grid with detergent ${ }^{17}$. The design of the chemistry was aimed at shielding the underlying carbon film with PEG groups, while preserving the holey geometry of the film. PEG is expected to be more hydrophilic than either plasma-cleaned amorphous carbon or gold film. Thus, we used surface wettability as a measure of SAM formation ${ }^{18}$. Upon applying a droplet of water, we observed from the drop contact angle that plasma-cleaned grids are more wettable than gold-coated grids that had undergone a mock reaction in pure ethanol, but grids with gold coating that had undergone reaction with thiol-PEG were the most wettable (Fig. 1). Moreover, the water was seen to equilibrate to both sides of the SAM grid by passing through the grid holes. This observed equilibration was attributed to the lower surface tension of the aqueous

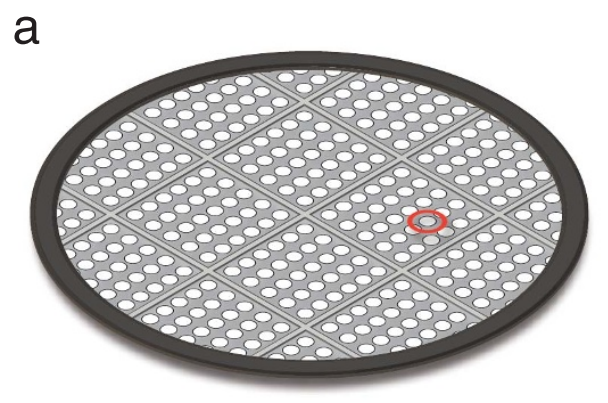

d

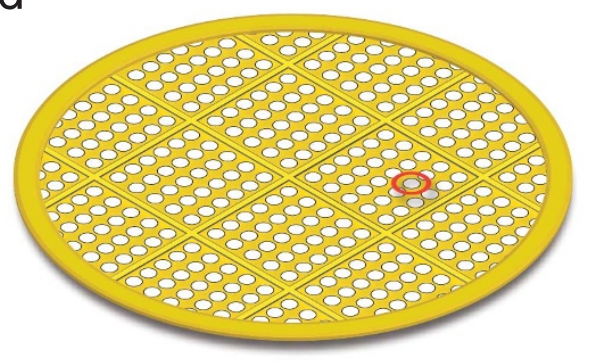

g

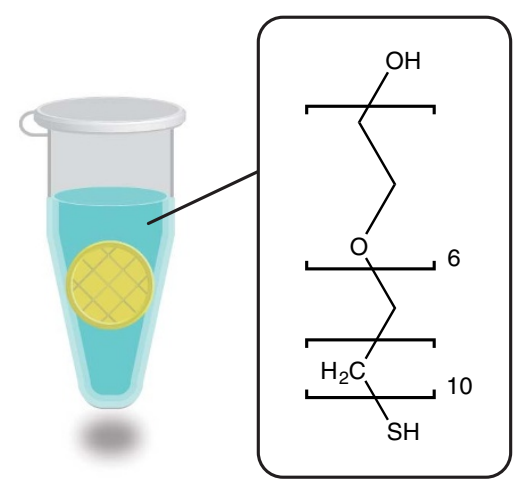

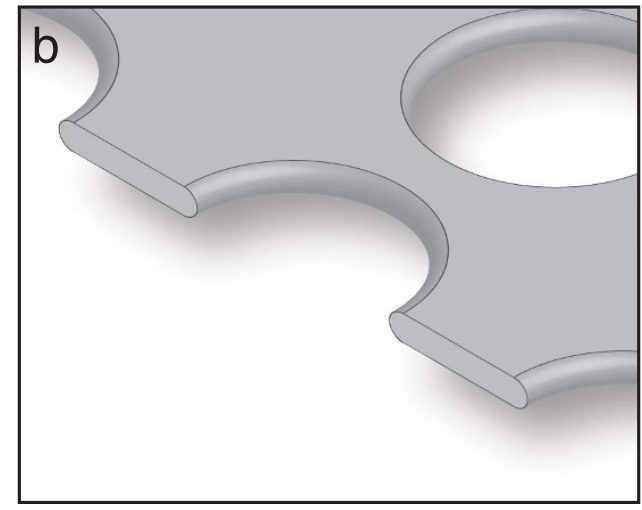
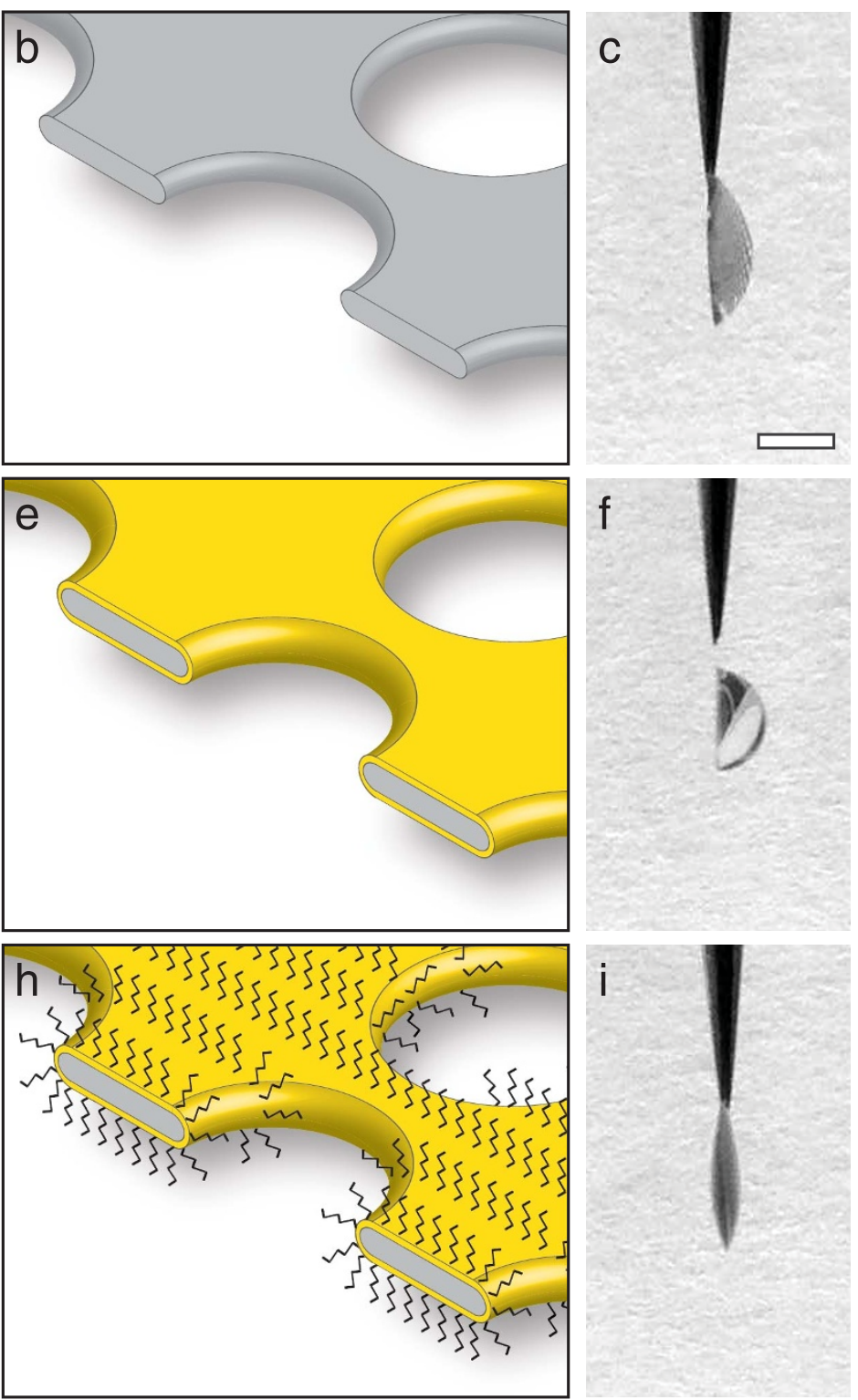

Figure 1 Preparation of SAM-grids and characterization of wettability. (a), (b). Schematic of holey carbon grid, with single hole highlighted in red (a) and shown in a cutaway view (b). (c). Wettability of carbon grid after plasma cleaning is good, as demonstrated by the acute contact angle. (d), (e). Illustrations of holey carbon grid after gold coating on both sides with single hole highlighted (d) and shown as a cutaway (e). (f). The wettability of goldcoated grid is decreased with respect to carbon grid as demonstrated by the high contact angle. (g), (h). Illustration of gold-coated grid undergoing reaction with PEG-thiol (g) to form SAM (h). (i). Surface wettability increased on SAM with respect to carbon and gold alone. In panels showing contact angle measurements ((c), (f), and (i)), the grid is held vertically by tweezers, and is viewed along its edge. Scale bar is $2 \mathrm{~mm}$. 

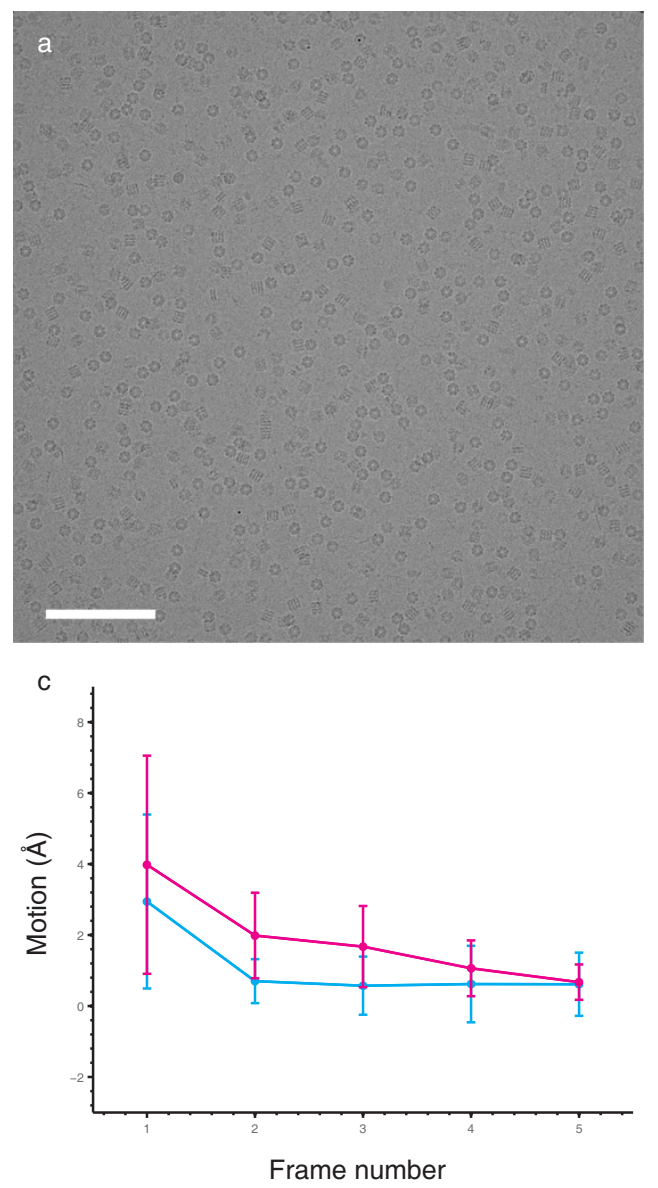
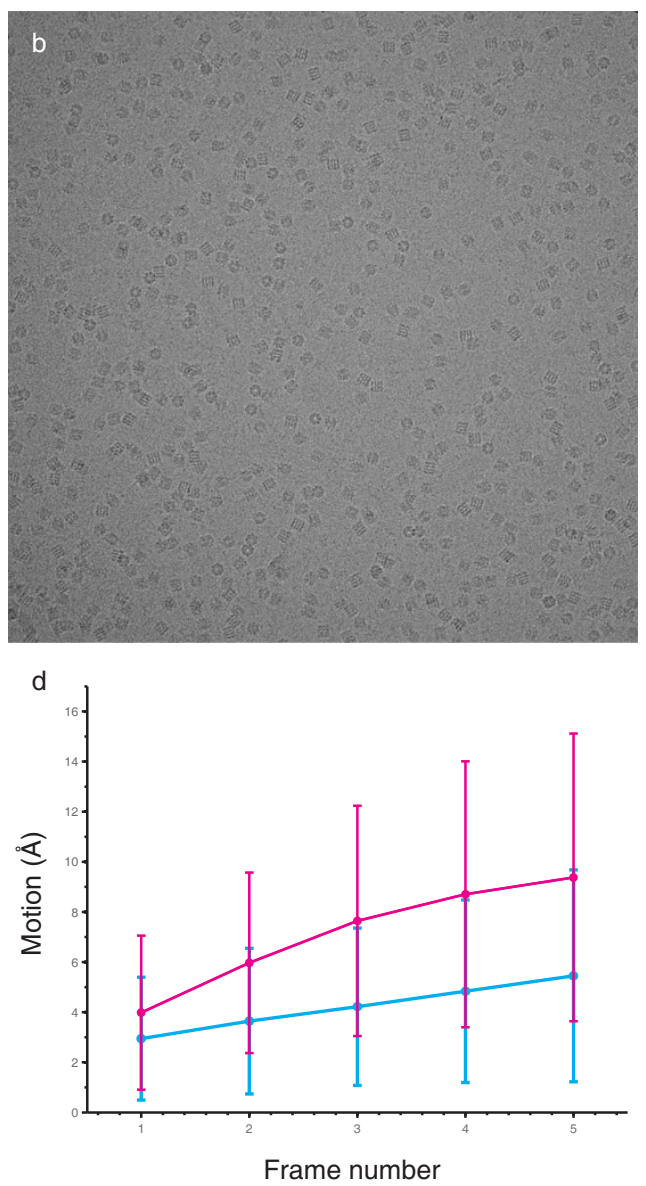

Figure 2 Comparison of motion in holes of carbon and SAM grids. (a) Image of GroEL on carbon grid. (b) Image of GroEL on SAM grid. (c) Average motion per frame. (d) Cumulative motion during exposure. Magenta curves are for carbon grid data $(N=735)$, blue curves are for SAM grid data $(N=1,986)$. Movie data was collected with seven frames, though frames are numbered from 1-5. This is because frame 0 is considered to be the alignment reference and thus has no associated shift. The final frame was taken at a higher total dose to aid in CTF estimation and was therefore omitted from motion analysis.

medium on the PEG surface, to the point that the water could spread through the holes in the support film. Gold film thickness was measured by ellipsometry to be $6.86 \mathrm{~nm}+/-0.042 \mathrm{~nm}$ per side. As such, when gold was deposited on both sides of the grid, the support thickness increased from $\sim 15 \mathrm{~nm}$ for carbon alone to $\sim 30 \mathrm{~nm}$ total thickness. The impact of the gold coating and the SAM on ice movement was assessed by recording movies of GroEL particles in holes of both unmodified holey carbon and SAM grids. In the present experiments we observed that the SAM grid showed $\sim 40 \%$ less beaminduced cumulative motion than that seen with conventional holey carbon supports over the course of a single low-dose exposure to the electron beam ( $5.45 \AA$ for SAM grids vs. $9.38 \AA$ for standard holey carbon grids, Fig. 2). Taken together, these results show that a PEGbased SAM can be introduced to the standard cryo-EM support to increase support hydrophilicity, while preserving support geometry. Although the modification roughly doubles the thickness of the support, this does not change the ability to obtain sufficiently thin films of vitrified ice suitable for imaging by cryo-EM.

We next tested whether the PEG-functionalized SAM grid would mitigate surface adhesion issues which prevented single particle imaging of GluK2 on standard holey carbon, and allow the particles to partition into the support holes. Our earlier analyses with GluK2 on conventional holey carbon supports had shown that in addition to being excluded from the holes, the proteins adsorbed on the carbon with a preferential orientation ${ }^{15}$. Images recorded from GluK2 specimens deposited on SAM-treated grids demonstrate that the modification yields particles in the holes (Fig. 3). This alteration in protein distribution was verified to hold for GluK2 receptor in closed (unpublished structure) and desensitized states, and the method was further extended to the related GluA2 AMPA receptor, where the use of SAM treatment enabled distribution of the protein into the holes in closed, active and desensitized states ${ }^{19}$. In each case, information on the $3 \mathrm{D}$ structure of the receptor could be obtained using single particle cryo-EM approaches, with the desensitized state of GluK2 resolved to 7.6 A (Fig. 3; EMDB: 2685), and the collection of GluA2 structures to between $10.4 \AA$ and $22.9 \AA^{19}$.

Our results show that gold-thiol self-assembled monolayer can be customized to modify the surface chemistry of the standard holey carbon support used in three-dimensional cryo-EM, and that the use of a hydrophilic SAM is a viable way to mitigate protein adhesion that could otherwise impede cryo-EM structure determination, as occurred during our initial analysis of GluK2 ${ }^{15}$. The method is simple and accessible to most cryo-EM laboratories, requiring only a sputter coater and a thiol reagent, both of which are commercially available at relatively low costs. Use of the SAM grid was essential to determining high resolution cryo-EM structures of kainate and AMPA receptors in distinct functional states by single particle reconstruction, which had previously not been feasible in our lab because of the strong preference of the protein to adsorb to the carbon surface.

\section{Discussion}

In this application we used a thiol displaying a PEG polymer which yields a hydrophilic surface. However, other functional groups can be 

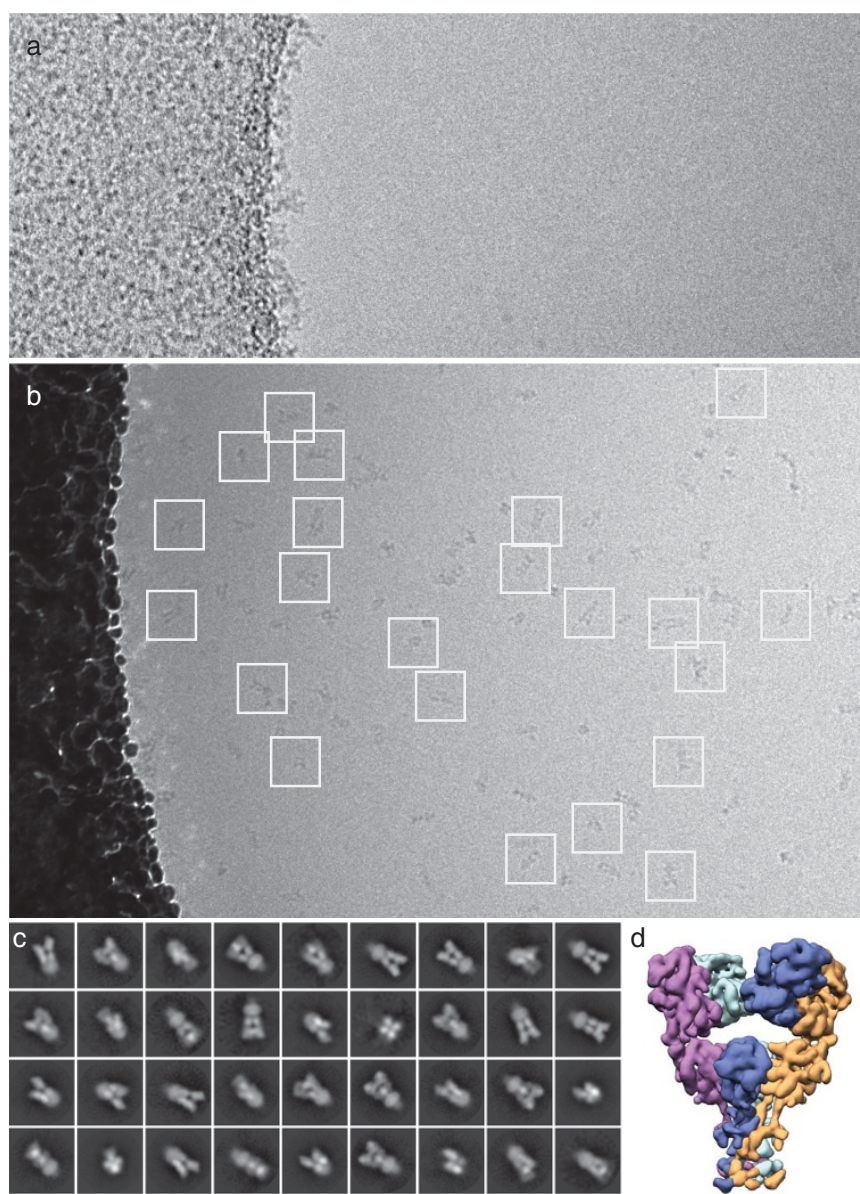

Figure 3 SAM-modified grids enable single particle imaging of GluK2. (a) Cryo-EM projection image of GluK2 on holey carbon grids showing that the protein has a strong preference for carbon film (dark gray, left) and is not available for imaging in the vitreous ice present in the holes (light gray, right). (b) Protein is observed in the holes of SAM grids prepared with PEG-thiol. Boxes highlight representative single particles. Scale bar is 50 $\mathrm{nm}$. (c, d) Subset of 2D classes of desensitized state GluK2 and resulting 3D reconstruction resolved to $7.6 \AA$ (EMDB: 2685) ${ }^{19}$.

displayed, so this approach can be used to achieve diverse surface chemistries (e.g. carboxylic acids for negative charge or amines for positive charge, at physiological $\mathrm{pH}$ ). We observed that the gold coating appears to reinforce the structural integrity of the holey film, and thereby diminish carbon breaking often observed following specimen blotting and mounting. This strengthening, in combination with the higher conductivity from the gold layer $(\sim 1,000$-fold higher than amorphous carbon), are likely origins of the reduction in beaminduced motion observed in the present experiments. Self-assembled monolayers thus offer an avenue to customize the surface chemistry of gold and to take advantage of the use of a thin holey metal support for high resolution cryo-EM analyses. This approach may enable structure determination of other macromolecular targets that suffer from high affinity for holey carbon, have a preferred orientation on continuous carbon, or are difficult to purify in large quantities such as many membrane proteins.

\section{Methods}

Self-assembled monolayer preparation. Quantifoil (Quantifoil, Jena, Germany) or C-flat (Protochips, Raleigh, NC, USA) holey carbon grids were cleaned for 6 seconds in a Solarus plasma cleaner (Gatan, Pleasantown, CA, USA). After cleaning, grids were gold sputter coated for 15 seconds on both sides using a Cressington 108 instrument (Cressington Scientific Instruments, Watford, UK). A self-assembly reaction mixture was prepared using ethanol as a solvent with $4 \mathrm{mM}$ monothiolalkane(C11)PEG6-OH (11-mercaptoundecyl) hexaethyleneglycol (SPT-
0011P6, SensoPath Technologies, Inc., Bozeman, MT, USA). Each gold-coated TEM grid was immersed in $100 \mathrm{uL}$ of reaction mixture contained in a $600 \mathrm{uL}$ snap-top conical tube. All reaction tubes were closed, sealed with Parafilm, and stored in a screw-top plastic jar filled with nitrogen gas. Reactions were allowed to proceed for 24-48 hours.

Cryo-EM specimen preparation. For experiments involving SAM grids, the grids were removed from the reaction mixture, dunked in ethanol to wash away unreacted thiol, and then air dried for 1-3 minutes to allow the ethanol to evaporate. For experiments involving un-modified holey carbon, the grids were plasma cleaned for 6 seconds in a Solarus plasma cleaner (Gatan, Pleasantown, CA, USA). Vitrified specimens were prepared by loading a grid into an FEI Mark IV Vitrobot (FEI Company, Hillsboro, OR, USA) adding $2.5 \mu \mathrm{L}$ of protein suspension to the grid, and then immediately blotting the grid for 2 seconds before plunge-freezing in liquid ethane $\left(\sim-180{ }^{\circ} \mathrm{C}\right)$. The Vitrobot environmental chamber was maintained at $22{ }^{\circ} \mathrm{C}$ and $100 \%$ humidity.

Cryo-electron microscopy and structure determination. Images shown in Figure 3 feature vitrified specimens of desensitized state GluK2, imaged using a Tecnai G2 Polara transmission electron microscope (FEI Company, Hillsboro, OR) operated at $200 \mathrm{kV}$, and equipped with an energy filter and $2 \mathrm{~K} \times 2 \mathrm{~K}$ post-energy filter CCD (Gatan, Pleasantown, CA). The specimens were maintained at a temperature of $\sim-180{ }^{\circ} \mathrm{C}$ throughout all steps of imaging. Images shown in Figure 3 were acquired at 34,000 $\times$ nominal magnification (effective pixel size of $4.1 \AA$ at the specimen plane) at $4 \mu \mathrm{m}$ underfocus and a dose of $\sim 20 \mathrm{e}^{-} / \AA^{2}$. Images used for $3 \mathrm{D}$ structure determination were acquired on an FEI Titan Krios electron microscope and processed as described in Meyerson et. al. 2014 ${ }^{19}$. The presence of gold on the grid did not impair auto-focusing routines used in data acquisition.

Protein preparation. Lyophilized GroEL (Cat \# C7688, Sigma-Aldrich, St. Louis, MO, USA) was solubilized in buffer containing $50 \mathrm{mM}$ TRIS pH 7.5, $10 \mathrm{mM} \mathrm{KCl}$ and $10 \mathrm{mM} \mathrm{MgCl}_{2}$. The solubilized protein was subjected to size-exclusion chromatography on a Superdex $20010 / 30$ column connected to a AKTA FPLC (GE, Pittsburgh, PA, USA). The elution volume fractions corresponding to the appropriate molecular weight of GroEL were pooled and concentrated using an Amicon (EMD Millipore, Billerica, MA, USA) concentrator with a 100,000 Da cut-off. The concentrated protein was thereafter added to an equal volume of pre-swelled Cibacron Blue 3G-A agarose (Cat \# B1064, Sigma-Aldrich, St. Louis, MO, USA) in batch-mode at $4{ }^{\circ} \mathrm{C}$ overnight. Centrifuging the suspension at $20,000 \times \mathrm{g}$ and collecting the supernatant recovered the GroEL. Protein concentration was measured as $2.5 \mathrm{mg} / \mathrm{mL}$ by monitoring the O.D.280 absorbance.

Homomeric rat GluK2 and GluA2 were expressed, purified and functionally trapped as described in Meyerson et. al. $2014^{19}$.

Support analysis. Gold film thickness measurements were carried out by gold sputter coating silicon wafers (Silicon Inc., Boise, ID, USA) for 15 seconds, and analyzing them in a M-2000D ellipsometer (J.A. Woollam, Lincoln, NE, USA). Interpretation of the ellipsometric data was done using gold on silicon oxide as a reference. A 15 second coating was measured to have a thickness of $6.86 \mathrm{~nm}+/-0.042 \mathrm{~nm}$.

The impact of the PEG SAM on support motion was assessed by imaging GroEL on Quantifoil R2/2 holey carbon, without and with the presence of PEG SAM having

$\sim 30 \mathrm{~nm}$ thickness (i.e. 15 second coating on both sides). GroEL grids were imaged as described for GluK2 and GluA2, but with focus values between $-3.0 \mu \mathrm{m}$ and

$-3.5 \mu \mathrm{m}$, a dose rate of $15 \mathrm{e}^{-} / \AA^{2} / \mathrm{s}$, and an exposure time of 4 seconds. For each seven frame movie, the first 6 frames were given $\sim 5 \mathrm{e}^{-} / \AA^{2}$, and frame 7 was given $\sim 30 \mathrm{e}^{-} /$ $\AA^{2}$. Unbinned frames were aligned using UCSF motioncorr ${ }^{20}$ and the resulting image shifts used to analyze motion.

1. Patwardhan, A. et al. Data management challenges in three-dimensional EM. Nat Struct Mol Biol 19, 1203-1207 (2012).

2. Bartesaghi, A., Matthies, D., Banerjee, S., Merk, A. \& Subramaniam, S. Structure of beta-galactosidase at 3.2-A resolution obtained by cryo-electron microscopy. Proc Natl Acad Sci U S A 111, 11709-11714 (2014).

3. Liao, M., Cao, E., Julius, D. \& Cheng, Y. Structure of the TRPV1 ion channel determined by electron cryo-microscopy. Nature 504, 107-112 (2013).

4. Dubochet, J. et al. Cryo-electron microscopy of vitrified specimens. Q Rev Biophys 21, 129-228 (1988).

5. Subramaniam, S., Gerstein, M., Oesterhelt, D. \& Henderson, R. Electron diffraction analysis of structural changes in the photocycle of bacteriorhodopsin. EMBO J 12, 1-8 (1993).

6. Pantelic, R. S., Suk, J. W., Hao, Y., Ruoff, R. S. \& Stahlberg, H. Oxidative doping renders graphene hydrophilic, facilitating its use as a support in biological TEM. Nano Lett 11, 4319-4323 (2011).

7. Rhinow, D. et al. Energy-filtered transmission electron microscopy of biological samples on highly transparent carbon nanomembranes. Ultramicroscopy 111, 342-349 (2011)

8. Russo, C. J. \& Passmore, L. A. Controlling protein adsorption on graphene for cryo-EM using low-energy hydrogen plasmas. Nat Methods 11, 649-652 (2014).

9. Yoshioka, C., Carragher, B. \& Potter, C. S. Cryomesh: a new substrate for cryoelectron microscopy. Microsc Microanal 16, 43-53 (2010). 
10. Rhinow, D. \& Kuhlbrandt, W. Electron cryo-microscopy of biological specimens on conductive titanium-silicon metal glass films. Ultramicroscopy 108, 698-705 (2008).

11. Jain, T., Sheehan, P., Crum, J., Carragher, B. \& Potter, C. S. Spotiton: a prototype for an integrated inkjet dispense and vitrification system for cryo-TEM. J Struct Biol 179, 68-75 (2012).

12. Liu, Y., Meng, X. \& Liu, Z. Deformed grids for single-particle cryo-electron microscopy of specimens exhibiting a preferred orientation. J Struct Biol 182, 255-258 (2013).

13. Kelly, D. F., Dukovski, D. \& Walz, T. A practical guide to the use of monolayer purification and affinity grids. Methods Enzymol 481, 83-107 (2010).

14. Quispe, J. et al. An improved holey carbon film for cryo-electron microscopy. Microsc Microanal 13, 365-371 (2007).

15. Schauder, D. M. et al. Glutamate receptor desensitization is mediated by changes in quaternary structure of the ligand binding domain. Proc Natl Acad Sci U S A (2013).

16. Prime, K. L. \& Whitesides, G. M. Self-assembled organic monolayers: model systems for studying adsorption of proteins at surfaces. Science 252, 1164-1167 (1991).

17. Cheung, M. et al. A method to achieve homogeneous dispersion of large transmembrane complexes within the holes of carbon films for electron cryomicroscopy. J Struct Biol (2013).

18. Suh, K. Y. \& Jon, S. Control over wettability of polyethylene glycol surfaces using capillary lithography. Langmuir 21, 6836-6841 (2005).

19. Meyerson, J. R. et al. Structural mechanism of glutamate receptor activation and desensitization. Nature 514, 328-334 (2014).
20. Li, X. et al. Electron counting and beam-induced motion correction enable nearatomic-resolution single-particle cryo-EM. Nat Methods 10, 584-590 (2013).

\section{Acknowledgments}

This work was supported by the intramural programs of the NCI, and NICHD, NIH, and IATAP program at NIH and the NIH-FEI Living Lab for Structural Biology. We thank Y.J. Eun for expert assistance with self-assembled monolayer design and chemistry, L. Earl for discussions on the manuscript, and S. Fellini, S. Chacko and their colleagues for continued support with use of the Biowulf cluster for computing at NIH.

\section{Author contributions}

J.R.M. and S.S. designed the method and experiments; J.R.M. analyzed the data; J.K. and S.C. carried out purification of glutamate receptors; S.B. carried out purification of GroEL; J.R.M., P.R. and J.P. carried out data collection; J.R.M., M.L.M. and S.S. interpreted the results and wrote the manuscript.

\section{Additional information}

Competing financial interests: The authors declare no competing financial interests.

How to cite this article: Meyerson, J.R. et al. Self-assembled monolayers improve protein distribution on holey carbon cryo-EM supports. Sci. Rep. 4, 7084; DOI:10.1038/srep07084 (2014).

(c) (i) (-) $\Theta$ This work is licensed under a Creative Commons Attribution-NonCommercialNoDerivs 4.0 International License. The images or other third party material in this article are included in the article's Creative Commons license, unless indicated otherwise in the credit line; if the material is not included under the Creative Commons license, users will need to obtain permission from the license holder in order to reproduce the material. To view a copy of this license, visit http:// creativecommons.org/licenses/by-nc-nd/4.0/ 